\title{
Semigroup Solution of Path-Dependent Second-Order Parabolic Partial Differential Equations
}

\author{
Sixian Jin and Henry Schellhorn \\ Claremont Graduate University, Claremont, CA, USA \\ Correspondence should be addressed to Henry Schellhorn; henry.schellhorn@cgu.edu
}

Received 16 December 2016; Accepted 1 February 2017; Published 27 February 2017

Academic Editor: Lukasz Stettner

Copyright (c) 2017 Sixian Jin and Henry Schellhorn. This is an open access article distributed under the Creative Commons Attribution License, which permits unrestricted use, distribution, and reproduction in any medium, provided the original work is properly cited.

\begin{abstract}
We apply a new series representation of martingales, developed by Malliavin calculus, to characterize the solution of the secondorder path-dependent partial differential equations (PDEs) of parabolic type. For instance, we show that the generator of the semigroup characterizing the solution of the path-dependent heat equation is equal to one-half times the second-order Malliavin derivative evaluated along the frozen path.
\end{abstract}

\section{Introduction}

In this paper we consider semilinear second-order pathdependent PDEs (PPDEs) of parabolic type. These equations were first introduced by Dupire [1] and Cont and Fournie [2] and will be defined properly in the next section.

To motivate our result, we first consider the heat equation expressed in terms of a backward time variable. For $t \leq T$ we look for a function $v(x, t)$ that solves

$$
\begin{aligned}
\frac{\partial v(x, t)}{\partial t}+\frac{1}{2} \frac{\partial^{2} v(x, t)}{\partial^{2} x} & =0 ; \\
v(x, T) & =\Psi(x) .
\end{aligned}
$$

It is well known (see, e.g., [3], chapter 9.2 or [4]) that the solution is given by the flow of the semigroup $S(t)$; that is, $v(\cdot, t)=S(t) \Psi$, where

$$
\begin{aligned}
S(t) \Psi(x) & =\exp \left(\frac{1}{2} \frac{\partial^{2}}{\partial x^{2}}(T-t)\right) \Psi(x) \\
& :=\sum_{i=0}^{\infty} \frac{(T-t)^{i}}{2^{i} i !} \frac{\partial^{2 i} \Psi(x)}{\partial x^{2 i}} .
\end{aligned}
$$

The differential operator $(1 / 2)\left(\partial^{2} / \partial x^{2}\right)$ is said to be the (infinitesimal) generator of the semigroup $S(t)$. Consider now the path-dependent version of the heat equation:

$$
\begin{aligned}
D_{t} v\left(x_{t}^{p}, t\right)+\frac{1}{2} D_{x x} v\left(x_{t}^{p}, t\right) & =0 ; \\
v\left(x_{T}^{p}, T\right) & =\Psi\left(x_{T}^{p}\right),
\end{aligned}
$$

where $x_{t}^{p}$ is a continuous path on the interval $[0, t]$ and the derivatives are Dupire's path derivatives. Our goal is to find the generator of the semigroup (flowing the solution) of PPDEs, which we will refer to as the semigroup of the PPDE. It turns out that $1 / 2 D_{x x}$, that is, one-half times the secondorder vertical derivative, is not the appropriate infinitesimal generator, because of path dependence. Indeed, the vertical derivative is the rate of change of the functional $v(\cdot, t)$ for a change at time $t$. The correct infinitesimal generator is equal to $(1 / 2) \omega^{t} \circ \mathbb{D}_{s}^{2}$, where $\mathbb{D}_{s}^{2}$ is the second-order Malliavin derivative of $F(\omega) \equiv \Psi\left(x_{T}^{p}(\omega)\right)$. An important difference is that $F$ is now viewed as a random variable, and the (first-order) Malliavin derivative is a stochastic process in 
the canonical probability space for Brownian motion. The stopping path operator $\omega^{t}$ was introduced in [5]. Informally, the action of the stopping path operator (which we define rigorously later) is to freeze the path after time $t$ :

$$
\omega^{t} \circ F(\omega)=F\left(\omega_{t}\right)
$$

where $\omega_{t}$ is the stopped path. The stopped Malliavin derivative $\omega^{t} \circ \mathbb{D}_{s}$ is thus an extension of both

(i) the Dupire derivative; while the Dupire derivative corresponds to changes of the path at only one time, the iterated derivatives $\omega^{t} \circ \mathbb{D}_{s_{1}, \ldots, s_{n}}^{n}$ are taken with respect to changes of the canonical path at many different times $s_{1}, \ldots, s_{n}$;

(ii) the Malliavin derivative; while the Dupire derivative can be taken pathwise, as far as we know, the construction of the Malliavin derivative necessitates the introduction of a probability space.

The proof of the representation result is straightforward. Let us consider the path-independent case (1). Let $B$ be Brownian motion. By Itô's lemma, it is obvious that $v(B(t), t)$ is a martingale, say $M_{t}$, and that the value of this martingale is the conditional expectation at time $t$ of $\Psi(B(T))$. Consider now a general path-dependent terminal condition $\Psi(B)$, in [5], Jin et al. gave a new representation of Brownian martingales $M_{t}$ (with $t \leq T$ ) as an exponential of a timedependent generator, applied to the terminal value $M_{T} \equiv$ $\Psi(B)$ :

$$
M_{t}=\exp \left(\frac{1}{2} \int_{t}^{T} \omega^{t} \circ \mathbb{D}_{s}^{2} \mathrm{~d} s\right) \Psi(B)
$$

By the functional Feynman-Kac formula introduced in $[1,6]$, it is immediate that $1 / 2 \omega^{t} \circ \mathbb{D}^{2}$ is the generator of the semigroup of the PPDE.

The main advantage of the semigroup method is that the solution of the PPDE can be constructed semianalytically: indeed, the method is similar to the Cauchy-Kowalewsky method, of calculating iteratively all the Malliavin derivatives of $\Psi$; (6) can be rewritten indeed as

$$
M_{t}=\sum_{i=0}^{\infty} \frac{1}{2^{i} i !} \int_{[t, T]^{i}} \omega^{t} \circ\left(\mathbb{D}_{s_{i}}^{2} \cdots \mathbb{D}_{s_{1}}^{2} \Psi(B)\right) \mathrm{d} s_{i} \cdots \mathrm{d} s_{1} .
$$

The main disadvantage can be seen immediately by considering (7): the terminal condition $\Psi$ must be infinitely Malliavin differentiable. In contradistinction, the viscosity solution given in [7] necessitates $\Psi$ to be only bounded and continuous. However, compared to the result shown in [6], $\Psi$ needs only to be defined on continuous paths.

This paper is composed of two parts. In the first part, we give a rigorous proof of the result (7). Indeed, we complete the proof of Theorem 2.3 in our article [5]; although the statement was correct in that paper, one step of the proof was not obvious to finish. In the second part we characterize the generator of the semilinear PPDE.

\section{Martingale Representation}

We first introduce some basic notations of Malliavin calculus. For a detailed introduction, we refer to [8] and our paper [5]. Let $\Omega=C([0, T], \mathbb{R})$ and $\left(\Omega, \mathscr{F},\left\{\mathscr{F}_{t}\right\}_{t \geq 0}, \mathbb{P}\right)$ be the complete filtered probability space, where the filtration $\left\{\mathscr{F}_{t}\right\}_{t \geq 0}$ is the usual augmentation of the filtration generated by Brownian motion $B$ on $\mathbb{R}$. The canonical Brownian motion can be also denoted by $B(t)=B(t, \omega)=\omega(t), t \in[0, T], \omega \in \Omega$, by emphasizing its sample path. We denote by $L^{2}(\mathbb{P})$ the space of square integrable random variables. For simplicity, we denote $(\mathrm{d} u)^{\otimes k}:=\mathrm{d} u_{1} \cdots \mathrm{d} u_{k}$.

We denote the Malliavin derivative of order $l$ at time $t_{1}, \ldots, t_{n}$ by $\mathbb{D}_{t_{1}, \ldots, t_{n}}^{l}$. We call $\mathbb{D}_{\infty}([0, T])$ the set of random variables which are infinitely Malliavin differentiable and $\mathscr{F}_{T}$-measurable, that is, for any integer $n$ and $F \in \mathbb{D}_{\infty}([0, T])$ :

$$
E\left[\left(\sup _{s_{1}, \ldots, s_{n} \in[0, T]}\left|\mathbb{D}_{s_{1}, \ldots, s_{n}}^{n} F\right|\right)^{2}\right]<+\infty
$$

Definition 1. For any deterministic function $f \in L^{2}([0, T])$, we define the "stopping path" operator $\omega^{t}$ for $t \leq T$ as

$$
\omega^{t} \circ \int_{0}^{T} f(s) \mathrm{d} B(s):=\int_{0}^{t} f(s) \mathrm{d} B(s)
$$

In particular, $\omega^{t} \circ B(s)=B(s \wedge t)$ that is to "freeze" Brownian motion after time $t$.

From the definition, it is not hard to obtain that, for any $n$ variable smooth function $g, \omega^{t} \circ g\left(B\left(s_{1}\right), \ldots, B\left(s_{n}\right)\right)=g\left(B\left(s_{1} \wedge\right.\right.$ $\left.t), \ldots, B\left(s_{n} \wedge t\right)\right)$. For a general random variable $F \in L^{2}(\mathbb{P})$, $\omega^{t} \circ F$ refers to the value of $F$ along the stopping scenario $\omega_{t} \equiv$ $\omega^{t}(\omega)$ of Brownian motion. According to the Wiener-Chaos decomposition, for any $F \in L^{2}(\mathbb{P})$, there exists a sequence of deterministic function $\left\{f_{n}\right\}_{n \geq 1}$ such that $F=\sum_{m=0}^{\infty} I_{m}\left(f_{m}\right)$ with convergence in $L^{2}\left([0, T]^{n}\right)$. Therefore, in order to obtain an explicit representation of $\omega^{t}$ acting on a general variable $F$, we first show the following proposition.

Proposition 2. Let $f_{n} \in L^{2}\left([0, T]^{n}\right)$, an $n$-variable square integrable deterministic function; then

$$
\begin{aligned}
& I_{n}\left(f_{n} \chi_{[0, t]}\right)=\omega^{t} \circ I_{n}\left(f_{n}\right) \\
& \quad+\sum_{k=1}^{\lfloor n / 2\rfloor} \frac{n !}{2^{k}(n-2 k) !} \int_{t \leq u_{1} \leq \cdots \leq u_{k} \leq T} \omega^{t} \\
& \quad \circ I_{n-2 k}\left(f_{n}\right)\left(u_{1}, u_{1}, \ldots, u_{k}, u_{k}\right)(\mathrm{d} u)^{\otimes k} .
\end{aligned}
$$


Therefore

$$
\omega^{t} \circ I_{n}\left(f_{n}\right)=n ! \sum_{k=0}^{\lfloor n / 2\rfloor} \frac{(-1)^{k}}{2^{k}(n-2 k) ! k !} \int_{[t, T]^{k}} \int_{[0, t]^{n-2 k}} f_{n}\left(s_{1}, \ldots, s_{n-2 k}, u_{1}, u_{1}, \ldots, u_{k}, u_{k}\right)\left(\mathrm{d} B_{s}\right)^{\otimes(n-2 k)}(\mathrm{d} u)^{\otimes k},
$$

as well as the isometry:

$$
E\left[\left(\omega^{t} \circ I_{n}\left(f_{n}\right)\right)^{2}\right]=n !^{2} \sum_{k=0}^{\lfloor n / 2\rfloor} \frac{1}{2^{2 k}((n-2 k) ! k !)^{2}} \int_{[t, T]^{k}} \int_{[0, t]^{n-2 k}} f_{n}\left(s_{1}, \ldots, s_{n-2 k}, u_{1}, u_{1}, \ldots, u_{k}, u_{k}\right)^{2}(\mathrm{~d} s)^{\otimes(n-2 k)}(\mathrm{d} u)^{\otimes k} .
$$

Theorem 3. Let $F \in L^{2}(\mathbb{P})$. Then for any fixed time $t$ and $t \leq s<T$, there exists a sequence $\left\{F^{N}\right\}_{N \geq 0}$ that satisfies the following:

(i) $F^{N} \rightarrow F$ in $L^{2}(\mathbb{P})$;

(ii) $D_{u} F^{N}=D_{s+1 / N} F^{N}$ for any $u \in(s, s+1 / N]$;

(iii) there exist $\varepsilon \in(0,1)$ and a constant $C$ which does not depend on $N$ such that

$$
E\left[\left(\omega^{t} \circ\left(F^{N}-F\right)\right)^{2}\right] \leq \frac{C}{N^{2+\varepsilon}} .
$$

We introduce the derivative $\mathrm{d}$ in $L^{2}(\mathbb{P})$ as, for any process $F_{s}$,

$$
G_{s}:=\frac{\mathrm{d} F_{s}}{\mathrm{~d} s}
$$

is defined by $\lim _{\varepsilon \rightarrow 0} E\left[\left(\frac{F_{s+\varepsilon}-F_{s}}{\varepsilon}-G_{s}\right)^{2}\right]=0$.

Then we can set up an operator differential equation for $E_{s}$. The following theorem is a generalization of Theorem 2.2. in [5] to functionals that are not discrete.

Theorem 4. For $0 \leq t \leq s \leq T$, assuming that $F \in \mathbb{D}^{6}([0, T])$, one has

$$
\frac{\mathrm{d} \omega^{t} \circ E\left[F \mid \mathscr{F}_{s}\right]}{\mathrm{d} s}=-\omega^{t} \circ \frac{1}{2} \mathbb{D}_{s}^{2} E\left[F \mid \mathscr{F}_{s}\right] .
$$

Then our main theorem is the integral version of this operator differential equation. We first introduce the convergence condition.

Condition 1. For any $n \geq 0, F$ satisfies

$$
\begin{aligned}
& \frac{(T-t)^{2 n}}{\left(2^{n} n !\right)^{2}} E\left[\left(\sup _{\substack{\longrightarrow \rightarrow \infty \\
u_{1}, \ldots, u_{n} \in[t, T]}}\left|\omega^{t} \circ \mathbb{D}_{u_{n}}^{2} \cdots \mathbb{D}_{u_{1}}^{2} F\right|\right)^{2}\right] \\
&
\end{aligned}
$$
$T])$.
Remark 5. We claim that other conditions exist which are easier to check than Condition 1. One of them is the convergence of the terms of series (23):

$$
\frac{(T-t)^{n}}{2^{n} n !} \sup _{u_{1}, \ldots, u_{n} \in[t, T]}\left|\omega^{t} \circ \mathbb{D}_{u_{n}}^{2} \cdots \mathbb{D}_{u_{1}}^{2} F\right| \underset{n \rightarrow \infty}{\longrightarrow} 0 \text { a.s. }
$$

To this "local" condition, that is, a condition based on the calculation along the frozen path only, one needs to add a "global" condition involving all the paths to make it sufficient; that is, $E\left[\left(\mathbb{D}_{s}^{n} F\right)^{2}\right]<c^{2 n}$ for any $s \in[t, T]$ and $n \geq 1$, with a constant $c$.

Moreover, with different structures of $F$, we have different alternative conditions which are easier to check for practical calculations. Here we list two examples.

(1) If $F=f\left(\int_{0}^{T} g(s) \mathrm{d} B(s)\right)$ with smooth deterministic function $f$ and square integrable deterministic function $g$, it is not hard to obtain

$$
\begin{aligned}
& \frac{(T-t)^{n}}{2^{n} n !} \sup _{u_{1}, \ldots, u_{n} \in[t, T]}\left|\omega^{t} \circ \mathbb{D}_{u_{n}}^{2} \cdots \mathbb{D}_{u_{1}}^{2} F\right| \\
& =\frac{(T-t)^{n}}{2^{n} n !}\left(\sup _{x \in[t, T]} g(x)\right)^{2 n} \\
& \cdot\left(f^{(2 n)}\left(\int_{0}^{t} g(s) \mathrm{d} B(s)\right)\right) .
\end{aligned}
$$

Therefore, if there exists a constant $C$ such that, for all $n \geq 1$,

$$
\left|\sup _{x \in \mathbb{R}} f^{(2 n)}(x)\right| \leq\left(\frac{n}{C}\right)^{2 n}
$$

with the help of Stirling approximation $n ! \sim \sqrt{2 \pi n}(n / e)^{n}$, Condition 1 is satisfied. 
(2) If $F$ has its chaos decomposition $F=\sum_{m=0}^{\infty} I_{m}\left(f_{m}\right)$, we have

$$
\begin{aligned}
\omega^{t} \circ & \mathbb{D}_{u_{n}}^{2} \cdots \mathbb{D}_{u_{1}}^{2} F \\
= & \sum_{m=2 n}^{\infty} \frac{m !}{(m-2 n) !} \omega^{t} \\
& \circ I_{m-2 n}\left(f_{m}\left(\cdot, u_{1}, u_{1}, \ldots, u_{n}, u_{n}\right)\right) .
\end{aligned}
$$

Then according to (12), Condition 1 can be replaced by

$$
\begin{aligned}
& \frac{C(T-t)^{2 n}}{\left(2^{n} n !\right)^{2}} \sum_{m=2 n}^{\infty}\left(\frac{m !}{(m-2 n) !}\right)^{2} \\
& \cdot \sup _{u_{1}, \ldots, u_{n} \in[t, T]} \int_{[0, t]^{m-2 n}} f_{m}\left(s_{1}, \ldots, s_{m-2 n}, u_{1}, u_{1}, \ldots, u_{n},\right. \\
& \left.u_{n}\right)^{2}(\mathrm{~d} s)^{\otimes m-2 n} \underset{n \rightarrow \infty}{\longrightarrow} 0,
\end{aligned}
$$

with some constant $C$ or some much stronger but easier conditions like the following: for $m \geq 1$

$$
\left|\sup _{s_{1}, \ldots, s_{m} \in[0, T]} f_{m}\left(s_{1}, \ldots, s_{m}\right)^{2}\right| \leq \frac{C}{m !} .
$$

Then we have the following main result.

Theorem 6. Suppose that $F$ satisfies Condition 1 and is $\mathscr{F}_{T^{-}}$ measurable. For $t \leq T$, then, in $L^{2}(\mathbb{P})$,

$$
E\left[F \mid \mathscr{F}_{t}\right]=\exp \left(\frac{1}{2} \int_{t}^{T} \omega^{t} \circ \mathbb{D}_{s}^{2} \mathrm{~d} s\right) F
$$

The importance of the exponential formula (23) stems from the Dyson series representation, which we rewrite hereafter in a more convenient way:

$$
\begin{aligned}
E\left[F \mid \mathscr{F}_{t}\right]= & \omega^{t} \circ F+\frac{1}{2} \int_{t}^{T} \omega^{t} \circ \mathbb{D}_{s}^{2} F \mathrm{~d} s \\
& +\frac{1}{4} \int_{t}^{T} \int_{s_{1}}^{T} \omega^{t} \circ \mathbb{D}_{s_{1}}^{2} \mathbb{D}_{s_{2}}^{2} F \mathrm{~d} s_{2} \mathrm{~d} s_{1}+\cdots .
\end{aligned}
$$

\section{Representation of Solutions of Path- Dependent Partial Differential Equations}

3.1. Functional Itô Calculus. We now introduce some key concepts of the functional Itô calculus introduced by Dupire [1]. For more information, the reader is referred to [6], which we copy hereafter almost verbatim. Let $T>0$ be fixed. For each $t \in[0, T]$ we denote by $\Lambda_{t}$ the set of càdlàg (right continuous with left limits) $\mathbb{R}$-valued functions on $[0, t]$. For each $\gamma_{t} \in \Lambda_{t}$, the value of $\gamma_{t}$ at $s \in[0, t]$ is denoted by $\gamma(s)$. Denote $\Lambda=\bigcup_{t \in[0, T]} \Lambda_{t}$. For each $\gamma_{t} \in \Lambda, T \geq s \geq t$, and $x \in \mathbb{R}$, we define

$$
\begin{aligned}
& \gamma_{t}^{x}(r):=\gamma(r) \mathbf{1}_{[0, t)}(r)+(\gamma(t)+x) \mathbf{1}_{\{t\}}(r), \\
& \quad r \in[0, t], \\
& \gamma_{t, s}(r):=\gamma(r) \mathbf{1}_{[0, t)}(r)+\gamma(t) \mathbf{1}_{[t, s]}(r), \quad r \in[0, s] .
\end{aligned}
$$

Definition 7. Given a function $\widehat{u}: \Lambda \rightarrow \mathbb{R}$, there exists $p \in \mathbb{R}$ such that

$$
\widehat{u}\left(\gamma_{t}^{x}\right)=\widehat{u}\left(\gamma_{t}\right)+p x+o(|x|) \quad \text { as } x \longrightarrow 0 .
$$

Then we say that $\widehat{u}$ is vertically differentiable at $\gamma_{t} \in \Lambda$ and define $D_{x} \widehat{u}\left(\gamma_{t}\right):=p$. The function $\widehat{u}$ is said to be vertically differentiable if $D_{x} \widehat{u}\left(\gamma_{t}\right)$ exists for each $\gamma_{t} \in \Lambda$. The secondorder derivative $D_{x x}$ is defined similarly.

Definition 8. For a given $\gamma_{t} \in \Lambda$, if

$$
\begin{aligned}
& \widehat{u}\left(\gamma_{t, s}\right)=\widehat{u}\left(\gamma_{t}\right)+a(s-t)+o(|s-t|) \\
& \text { as } s \longrightarrow t, s \geq t,
\end{aligned}
$$

then we say that $\widehat{u}$ is horizontally differentiable at $\gamma_{t}$ and define $D_{t} \widehat{u}\left(\gamma_{t}\right):=a$. The function $\widehat{u}$ is said to be horizontally differentiable if $D_{x} \widehat{u}\left(\gamma_{t}\right)$ exists for each $\gamma_{t} \in \Lambda$.

Definition 9. The function $\widehat{u}$ is said to be in $\mathbb{C}_{l, \text { Lip }}^{1,2}(\Lambda)$ if $D_{t} \widehat{u}$, $D_{x} \widehat{u}$, and $D_{x x} \widehat{u}$ exist and we have

$$
\begin{array}{r}
\left|\varphi\left(\gamma_{t}\right)-\varphi\left(\bar{\gamma}_{\bar{t}}\right)\right| \leq C\left(1+\left\|\gamma_{t}\right\|^{k}+\left\|\bar{\gamma}_{\bar{t}}\right\|^{k}\right) d_{\infty}\left(\gamma_{t}, \bar{\gamma}_{\bar{t}}\right) \\
\text { for each } \gamma_{t}, \bar{\gamma}_{\bar{t}} \in \Lambda,
\end{array}
$$

where $\varphi=\widehat{u}, D_{t} \widehat{u}, D_{x} \widehat{u}, D_{x x} \widehat{u}, C$ and $k$ are some constants depending only on $\varphi$, and

$$
d_{\infty}\left(\gamma_{t}, \bar{\gamma}_{\bar{t}}\right):=\sup _{s \in[0, t \vee \bar{t}]}|\gamma(s \wedge t)-\bar{\gamma}(s \wedge \bar{t})|+|t-\bar{t}|^{1 / 2}
$$

is the distance on $\Lambda$. The classes $\mathbb{C}_{l, \text { Lip }}^{0,1}$ and $\mathbb{C}_{l, \text { Lip }}^{0,2}$ are defined analogously.

For each $t \in[0, T]$, we denote by $\Omega_{t}$ the set of continuous $\mathbb{R}$-valued functions on $[0, t]$. We denote $\Omega=\bigcup_{t \in[0, T]} \Omega_{t}$. Clearly $\Omega \subseteq \Lambda$. Given $\widehat{u}: \Lambda \rightarrow \mathbb{R}$ and $u: \Omega \rightarrow \mathbb{R}$, we say that $u$ is consistent with $\widehat{u}$ on $\Omega$ if (since we already use the symbol $\omega^{t}$ to denote our freezing path operator (see Definition 1), we here use $\omega_{t}$ to denote a sample path) for each $\omega_{t} \in \Omega$,

$$
u\left(\omega_{t}\right)=\widehat{u}\left(\omega_{t}\right) .
$$

Definition 10. The function $u: \Omega \rightarrow \mathbb{R}$ is said to be in $\mathbb{C}_{l, \text { Lip }}^{1,2}(\Omega)$ if there exists a function $\widehat{u} \in \mathbb{C}_{l, \text { Lip }}^{1,2}(\Lambda)$ such that $(30)$ holds and for $\omega_{t} \in \Omega$ we denote

$$
\begin{aligned}
D_{t} u\left(\omega_{t}\right) & =D_{t} \widehat{u}\left(\omega_{t}\right), \\
D_{x} u\left(\omega_{t}\right) & =D_{x} \widehat{u}\left(\omega_{t}\right), \\
D_{x x} u\left(\omega_{t}\right) & =D_{x x} \widehat{u}\left(\omega_{t}\right) .
\end{aligned}
$$

Note. In the introduction, we use the notation $\{v(\cdot, t)\}$ for a family of nonanticipative functionals where $v(\cdot, t): \Lambda_{t} \rightarrow$ $\mathbb{R}$. In order to highlight the symmetry between PDEs and PPDEs, the notation $v\left(x_{t}^{p}, t\right)$ in PPDEs shows that $x_{t}^{p}$ is the counterpart of the argument $x$ in PDEs and is used instead of $\omega_{t}$. This is in spirit closer to the original notation of $[1,2]$. The reader will have no problem identifying $u\left(x_{t}^{p}\right)=v\left(x_{t}^{p}, t\right)$. 
3.2. Non-Markovian BSDEs. As in [6], we use $\mathscr{F}_{r}^{t}$ to denote the completion of the $\sigma$-algebra generated by $B(s)-B(t)$ with $s \in[t, r]$. Then we introduce $\mathscr{H}^{2}(t, T)$, the space of all $\mathscr{F}_{s}^{t}$-adapted $\mathbb{R}$-valued processes $(X(s))_{s \in[t, T]}$ with $E\left[\int_{t}^{T}|X(s)|^{2} \mathrm{~d} s\right]<\infty$, and $S^{2}(t, T)$, the space of all $\mathscr{F}_{s^{-}}$ adapted $\mathbb{R}$-valued continuous processes $(X(s))_{s \in[t, T]}$ with $E\left[\sup _{s \in[t, T]}|X(s)|^{2}\right]<\infty$. Denote now $\gamma_{\gamma_{t}^{x}}(r)=\gamma(r) \mathbf{1}_{[0, t)}(r)+$ $(\gamma(r)+x) \mathbf{1}_{[t, T]}(r)$.

We will make the following assumptions:

(H1) $\Phi$ is a $\mathbb{R}$-valued function defined on $\Lambda_{T}$. Moreover, $\Phi \in \mathbb{C}_{l, \mathrm{Lip}}^{1,2}\left(\Lambda_{T}\right)$.

(H2) The drift $a\left(\gamma_{t}\right)$ is a given $\mathbb{R}$-valued continuous function defined on $\Lambda$ (see [6] for a definition of continuity). For any $\gamma_{t} \in \Lambda$ and $s \in[0, t]$, the function $x \rightarrow a\left(\left(\gamma_{t}\right)_{\gamma_{s}^{x}}\right)$ is differentiable and its derivative $\mathrm{d} a\left(\left(\gamma_{t}\right)_{\gamma_{s}^{x}}\right) / \mathrm{d} x:=\varphi(x)$ satisfies

$$
\begin{aligned}
&|\varphi(x)-\varphi(y)| \leq C\left(1+|x|^{k}+|y|^{k}\right)|x-y|, \\
& \forall x, y \in \mathbb{R},
\end{aligned}
$$

where $C$ and $k$ are constants depending only on $\varphi$.

We now assume that (H1) and (H2) hold. We consider a non-Markovian BSDE, which is a particular case of (3.2) in [6]. From Theorem 2.8 in [6], for any $\gamma_{t} \in \Lambda$, there exists a unique solution $\left(Y_{\gamma_{t}}(s), Z_{\gamma_{t}}(s)\right)_{t \leq s \leq T} \in S^{2}(t, T) \times \mathscr{H}^{2}(t, T)$ of the following BSDE:

$$
\begin{aligned}
Y_{\gamma_{t}}(s)= & \Phi\left(B^{\gamma_{t}}\right)+\int_{s}^{T} a\left(B_{r}^{\gamma_{t}}\right) Y_{\gamma_{t}}(r) \mathrm{d} r \\
& -\int_{s}^{T} Z_{\gamma_{t}}(r) \mathrm{d} B(r),
\end{aligned}
$$

where

$$
\begin{aligned}
B^{\gamma_{t}}(u):= & \gamma(u) \mathbf{1}_{[0, t)}(u) \\
& +(\gamma(t)+B(u)-B(t)) \mathbf{1}_{(t, T]}(u)
\end{aligned}
$$

In particular, $Y_{\gamma_{t}}(t)$ defines a deterministic mapping from $\Lambda$ to $\mathbb{R}$.

3.3. Path-Dependent PDEs. The drift $a$ and terminal condition $\Psi$ are required to be extended to the space of càdlàg paths because of the definition of the Dupire derivatives. We require the following (see [6] again):

(B1) The function $\Psi$ is a $\mathbb{R}$-valued function defined on $\Omega_{T}$. Moreover, there is a function $\Phi \in \mathbb{C}_{l, \mathrm{Lip}}^{1,2}\left(\Lambda_{T}\right)$ such that $\Psi=\Phi$ on $\Omega_{T}$.

(B2) The drift $a\left(\omega_{t}\right)$ is a given $\mathbb{R}$-valued continuous function defined on $\Omega \times \mathbb{R} \times \mathbb{R}$ (see [6] for a definition of continuity). Moreover, there exists a function $b$ satisfying (H2) such that $a=b$ on $\Omega$.
We can now define the following quasilinear parabolic path-dependent PDE:

$$
\begin{aligned}
D_{t} u\left(\omega_{t}\right)+a\left(\omega_{t}\right) D_{x} u\left(\omega_{t}\right)+\frac{1}{2} D_{x x} u\left(\omega_{t}\right) & =0, \\
\omega_{t} \in \Omega, & t \in[0, T) ; \\
u\left(\omega_{T}\right) & =\Psi\left(\omega_{T}\right), \\
& \omega_{T} \in \Omega_{T} .
\end{aligned}
$$

Theorem 4.2 in [6] states the following: let $u \in C_{l, \text { Lip }}^{1,2}(\Omega)$ be a solution of the above equation. Then we have $u\left(\omega_{t}\right)=$ $Y_{\omega_{t}}(t)$ for each $\omega_{t} \in \Omega$, where $\left(Y_{\omega_{t}}(s), Z_{\omega_{t}}(s)\right)_{t \leq s \leq T}$ is the unique solution of BSDE (33).

Theorem 11. Suppose that, for each $t \in[0, T]$, the random variable

$$
F \equiv \exp \left(\int_{t}^{T} a\left(B^{\omega_{t}}(r)\right) \mathrm{d} r\right) \Psi\left(B^{\omega_{t}}\right)
$$

satisfies Condition 1. Then the solution of (35) is

$$
u\left(\omega_{t}\right)=\exp \left(\frac{1}{2} \int_{t}^{T} \omega^{t} \circ \mathbb{D}_{u}^{2} \mathrm{~d} u\right) F
$$

Proof. According to (2.20) in [9] page 351, the solution of (33) is, for $t \leq s \leq T$,

$$
\widehat{Y}_{\omega_{t}}(s)=E\left[\exp \left(\int_{s}^{T} a\left(B^{\omega_{t}}(r)\right) \mathrm{d} r\right) \Phi\left(B^{\omega_{t}}\right) \mid \mathscr{F}_{s}\right] .
$$

The result now follows by Theorem 6 and the fact that $u\left(\omega_{t}\right)=$ $Y_{\omega_{t}}(t)$.

We note that, in the case of no drift $(a=0)$, we recover the result (6).

3.4. Proof of Proposition 2. This proof is made up by several inductions. Therefore we separate them into several steps.

Step 1. We first apply Itô's lemma and integration by parts formula of the Skorohod integral of Brownian motion to provide an explicit expansion for $I_{n}\left(f_{n}\right)$. The goal of the following step is to transform Skorohod integrals into timeintegrals. For example, $f\left(s_{1}, s_{2}\right)$ is symmetric:

$$
\begin{aligned}
& I_{2}(f)=\int_{0}^{T} \int_{0}^{T} f\left(s_{1}, s_{2}\right) \mathrm{d} B\left(s_{2}\right) \mathrm{d} B\left(s_{1}\right) \\
& =\int_{0}^{T}\left(B(T) f\left(s_{1}, T\right)\right. \\
& \left.-\int_{0}^{T} B\left(s_{2}\right) f_{s_{2}}\left(s_{1}, s_{2}\right) \mathrm{d} s_{2}\right) \mathrm{d} B\left(s_{1}\right) .
\end{aligned}
$$


By the integration by parts formula (see (1.49) in [8]),

$$
\begin{aligned}
& I_{2}(f)=B(T) \int_{0}^{T} f\left(s_{1}, T\right) \mathrm{d} B\left(s_{1}\right)-\int_{0}^{T} f\left(s_{1}, T\right) \mathrm{d} s_{1} \\
& \quad-\int_{0}^{T}\left(B\left(s_{2}\right) \int_{0}^{T} f_{s_{2}}\left(s_{1}, s_{2}\right) \mathrm{d} B\left(s_{1}\right)\right. \\
& \left.\quad-\int_{0}^{s_{2}} f_{s_{2}}\left(s_{1}, s_{2}\right) \mathrm{d} s_{1}\right) \mathrm{d} s_{2}=B(T)^{2} f(T, T) \\
& \quad-B(T) \int_{0}^{T} f_{s_{1}}\left(s_{1}, T\right) B\left(s_{1}\right) \mathrm{d} s_{1}-\int_{0}^{T} f\left(s_{1}, T\right) \mathrm{d} s_{1} \\
& \quad-\int_{0}^{T} B\left(s_{2}\right) B(T) f_{s_{2}}\left(T, s_{2}\right) \mathrm{d} s_{2} \\
& \quad+\int_{0}^{T} \int_{0}^{T} B\left(s_{1}\right) B\left(s_{2}\right) f_{s_{1} s_{2}}\left(s_{1}, s_{2}\right) \mathrm{d} s_{1} \mathrm{~d} s_{2} \\
& \quad+\int_{0}^{T} \int_{0}^{s_{2}} f_{s_{2}}\left(s_{1}, s_{2}\right) \mathrm{d} s_{1} \mathrm{~d} s_{2}=\left(B(T)^{2} f(T, T)\right. \\
& \quad-2 B(T) \int_{0}^{T} f_{s_{1}}\left(s_{1}, T\right) B\left(s_{1}\right) \mathrm{d} s_{1} \\
& \left.\quad+\int_{0}^{T} \int_{0}^{T} B\left(s_{1}\right) B\left(s_{2}\right) f_{s_{1} s_{2}}\left(s_{1}, s_{2}\right) \mathrm{d} s_{1} \mathrm{~d} s_{2}\right) \\
& \quad-\int_{0}^{T} f(u, u) \mathrm{d} u .
\end{aligned}
$$

based on the following recurrence formula of $A_{r}$ : for any $r=$ $0, \ldots, n-1$

$$
\begin{aligned}
\int_{0}^{T} & A_{r}^{T}\left(s_{r+1}, \ldots, s_{n}\right) \mathrm{d} B\left(s_{r+1}\right) \\
= & A_{r+1}^{T}\left(s_{r+2}, \ldots, s_{n}\right) \\
& \quad-r \int_{0}^{T} A_{r-1}^{T}\left(u, u, s_{r+2}, \ldots, s_{n}\right) \mathrm{d} u .
\end{aligned}
$$

To prove (43), we apply the integration by parts formula. For simplicity, we only keep the variables $s_{1}, \ldots, s_{k}$ and $s_{r+1}$. The notation $\hat{x}$ means that the variable $x$ is not an argument of a function. We also emphasize again the symmetricity of function $f_{n}$ :

$$
\begin{aligned}
& \int_{0}^{T} A_{r}^{T}\left(s_{r+1}\right) \mathrm{d} B\left(s_{r+1}\right)=\sum_{k=0}^{r}(-1)^{k}\left(\begin{array}{l}
r \\
k
\end{array}\right) \int_{[0, T]^{k}}\left(\int_{0}^{T} \frac{\partial f_{n}\left(s_{1}, \ldots, s_{k}, s_{r+1}\right)}{\partial s_{1} \cdots \partial s_{k}} B(T)^{r-k} B\left(s_{1}\right) \cdots B\left(s_{k}\right) \mathrm{d} B\left(s_{r+1}\right)\right)(\mathrm{d} s)^{\otimes k} \\
& \quad=\sum_{k=0}^{r}(-1)^{k}\left(\begin{array}{l}
r \\
k
\end{array}\right)\left\{\int_{[0, T]^{k}} B(T)^{r-k} B\left(s_{1}\right) \cdots B\left(s_{k}\right) \int_{0}^{T} \frac{\partial f_{n}\left(s_{1}, \ldots, s_{k}, s_{r+1}\right)}{\partial s_{1} \cdots \partial s_{k}} \mathrm{~d} B\left(s_{r+1}\right)(\mathrm{d} s)^{\otimes k}\right. \\
& \quad-\int_{[0, T]^{k}} \sum_{i=1}^{k} B(T)^{r-k} \int_{0}^{s_{i}} B\left(s_{1}\right) \cdots \widehat{B}\left(s_{i}\right) \cdots B\left(s_{k}\right) B\left(s_{r+1}\right) \frac{\partial f_{n}\left(s_{1}, \ldots, s_{k}, s_{r+1}\right)}{\partial s_{1} \cdots \partial s_{k}} \mathrm{~d} s_{r+1}(\mathrm{~d} s)^{\otimes k} \\
& \left.-\int_{[0, T]^{k}}(n-k) B(T)^{r-k-1} \int_{0}^{T} B\left(s_{1}\right) \cdots B\left(s_{k}\right) \frac{\partial f_{n}\left(s_{1}, \ldots, s_{k}, s_{r+1}\right)}{\partial s_{1} \cdots \partial s_{k} \partial s_{r+1}} \mathrm{~d} s_{r+1}(\mathrm{~d} s)^{\otimes k}\right\} \\
& =\sum_{k=0}^{r}(-1)^{k}\left(\begin{array}{l}
r \\
k
\end{array}\right)\left\{\int_{[0, T]^{k}} B(T)^{r-k+1} B\left(s_{1}\right) \cdots B\left(s_{k}\right) \frac{\partial f_{n}\left(s_{1}, \ldots, s_{k}, T\right)}{\partial s_{1} \cdots \partial s_{k}}(\mathrm{~d} s)^{\otimes k}\right. \\
& -\int_{[0, T]^{k+1}} B(T)^{r-k} B\left(s_{1}\right) \cdots B\left(s_{k}\right) B\left(s_{r+1}\right) \frac{\partial f_{n}\left(s_{1}, \ldots, s_{k}, s_{r+1}\right)}{\partial s_{1} \cdots \partial s_{k} \partial s_{r+1}} \mathrm{~d} s_{r+1}(\mathrm{~d} s)^{\otimes k}
\end{aligned}
$$




$$
\begin{aligned}
& -\int_{[0, T]^{k}} k B(T)^{r-k} B\left(s_{1}\right) \cdots B\left(s_{k-1}\right) \frac{\partial f_{n}\left(s_{1}, \ldots, s_{k-1}, s_{k}, T\right)}{\partial s_{1} \cdots \partial s_{k-1}} \mathrm{~d} s_{k}(\mathrm{~d} s)^{\otimes k-1} \\
& +\int_{[0, T]^{k-1}} \int_{0}^{T} k B(T)^{r-k} B\left(s_{1}\right) \cdots B\left(s_{k-1}\right) \frac{\partial f_{n}\left(s_{1}, \ldots, s_{k-1}, u, u\right)}{\partial s_{1} \cdots \partial s_{k-1}}(\mathrm{~d} s)^{\otimes k-1} \mathrm{~d} u \\
& \left.-\int_{[0, T]^{k+1}}(n-k) B(T)^{r-k-1} B\left(s_{1}\right) \cdots B\left(s_{k}\right) \frac{\partial f_{n}\left(s_{1}, \ldots, s_{k}, s_{r+1}, T\right)}{\partial s_{1} \cdots \partial s_{k}} \mathrm{~d} s_{r+1}(\mathrm{~d} s)^{\otimes k}\right\} .
\end{aligned}
$$

Observing the properties of the binomial coefficients,

$$
\begin{aligned}
\left(\begin{array}{c}
r \\
k+1
\end{array}\right)(k+1)-\left(\begin{array}{l}
r \\
k
\end{array}\right)(r-k) & =0 ; \\
\left(\begin{array}{l}
r \\
k
\end{array}\right)+\left(\begin{array}{c}
r \\
k+1
\end{array}\right) & =\left(\begin{array}{l}
r+1 \\
k+1
\end{array}\right) ; \\
\left(\begin{array}{l}
r \\
k
\end{array}\right) k & =r\left(\begin{array}{l}
r-1 \\
k-1
\end{array}\right) .
\end{aligned}
$$

We can see that, under the summation over $k$, (47) and (49) cancel each other, (45) and (46) combine into $A_{r+1}^{T}$, and (48) remains as the integral of $A_{r-1}^{T}$. Rigorously, we proved (43).

To prove (42), we use induction. Supposing that case $n$ is correct, we observe case $n+1$ : by (43),

$$
\begin{aligned}
& \int_{0}^{T} I_{n}\left(f_{n}\right)\left(s_{n+1}\right) \mathrm{d} B\left(s_{n+1}\right)=\sum_{k=0}^{\lfloor n / 2\rfloor} \frac{(-1)^{k} n !}{2^{k}(n-2 k) ! k !} \\
& \cdot \int_{[0, T]^{k}} \int_{0}^{T} A_{n-2 k}^{T}\left(s_{n+1}, u_{1}, u_{1}, \ldots, u_{k}, u_{k}\right) \mathrm{d} B\left(s_{n+1}\right)(\mathrm{d} u)^{\otimes k} \\
& =\sum_{k=0}^{\lfloor n / 2\rfloor} \frac{(-1)^{k} n !}{2^{k}(n-2 k) ! k !} \int_{[0, T]^{k}}\left(A_{n+1-2 k}^{T}\left(u_{1}, u_{1}, \ldots, u_{k}, u_{k}\right)\right. \\
& \left.-(n-2 k) \int_{0}^{T} A_{n-1-2 k}^{T}\left(u_{1}, u_{1}, \ldots, u_{k}, u_{k}, u_{k+1}, u_{k+1}\right) \mathrm{d} u_{k+1}\right)(\mathrm{d} u)^{\otimes k} \\
& =\sum_{k=0}^{\lfloor(n+1) / 2\rfloor} \frac{(-1)^{k}(n+1) !}{2^{k}(n+1-2 k) ! k !} \\
& \cdot \int_{[0, T]^{k}} \int_{0}^{T} A_{n+1-2 k}^{T}\left(s_{n+1}, u_{1}, u_{1}, \ldots, u_{k}, u_{k}\right) \mathrm{d} B\left(s_{n+1}\right)(\mathrm{d} u)^{\otimes k} .
\end{aligned}
$$

Step 2. Now we are going to consider the action of the freezing path operator. We first prove that for all $r \leq n$

$$
\omega^{t} \circ A_{r}^{T}\left(s_{r+1}, \ldots, s_{n}\right)=A_{r}^{t}\left(s_{r+1}, \ldots, s_{n}\right) .
$$

We only present the proof of $r=n$ and the general case is the same. By definition, we know that $\omega^{t} \circ B_{s}=B_{s} \chi_{[0, t]}(s)+$ $B_{t} \chi_{[t, T]}(s)$. Therefore

$$
\begin{aligned}
\omega^{t} \circ A_{n}^{T} & =\sum_{k=0}^{n}(-1)^{k} \\
\cdot\left(\begin{array}{l}
n \\
k
\end{array}\right) & \int_{[0, T]^{k}} \frac{\partial f_{n}\left(s_{1}, \ldots, s_{k}, T, \ldots, T\right)}{\partial s_{1} \cdots \partial s_{k}} B(t)^{n-k} \omega^{t}
\end{aligned}
$$

$$
\begin{aligned}
& \circ\left(B\left(s_{1}\right) \cdots B\left(s_{k}\right)\right)(\mathrm{d} s)^{\otimes k}=\sum_{k=0}^{n}(-1)^{k} \\
& \cdot\left(\begin{array}{l}
n \\
k
\end{array}\right) \int_{[0, T]^{k}} \frac{\partial f_{n}\left(s_{1}, \ldots, s_{k}, T, \ldots, T\right)}{\partial s_{1} \cdots \partial s_{k}} B(t)^{n-k} \\
& \cdot \prod_{i=1}^{k}\left(B\left(s_{i}\right) \chi_{[0, t]}\left(s_{i}\right)+B(t) \chi_{[t, T]}\left(s_{i}\right)\right)(\mathrm{d} s)^{\otimes k} \\
& =\sum_{k=0}^{n}(-1)^{k}\left(\begin{array}{l}
n \\
k
\end{array}\right) \sum_{k_{1}=0}^{k}\left(\begin{array}{c}
k \\
k_{1}
\end{array}\right) \\
& \cdot \int_{[0, t]^{k_{1}} \times[t, T]^{k-k_{1}}} \frac{\partial f_{n}\left(s_{1}, \ldots, s_{k}, T, \ldots, T\right)}{\partial s_{1} \cdots \partial s_{k}} B(t)^{n-k_{1}} \\
& \cdot B\left(s_{1}\right) \cdots B\left(s_{k_{1}}\right)(\mathrm{d} s)^{\otimes k} .
\end{aligned}
$$

Now we recall a basic integration rule for a smooth function $g_{n}$ as

$$
\begin{aligned}
& \int_{[t, T]^{n}} \frac{\partial g_{n}\left(s_{1}, \ldots, s_{n}\right)}{\partial s_{1} \cdots \partial s_{n}}(\mathrm{~d} s)^{\otimes k} \\
& \quad=\sum_{j=0}^{n}(-1)^{j}\left(\begin{array}{c}
n \\
j
\end{array}\right) g_{n}(\underbrace{T, \ldots, T}_{n-j}, \underbrace{t, \ldots, t}_{j}) .
\end{aligned}
$$

We apply (54) on (53) and obtain

$$
\begin{gathered}
\omega^{t} \circ A_{n}^{T}=\sum_{k=0}^{n} \sum_{k_{1}=0}^{k} \sum_{j=0}^{k-k_{1}}(-1)^{k+j}\left(\begin{array}{l}
n \\
k
\end{array}\right)\left(\begin{array}{c}
k \\
k_{1}
\end{array}\right)\left(\begin{array}{c}
k-k_{1} \\
j
\end{array}\right) \\
\cdot \int_{[0, t]^{k_{1}}} \frac{\partial f_{n}}{\partial s_{1} \cdots \partial s_{k}}(s_{1}, \ldots, s_{k_{1}}, \underbrace{T, \ldots, T}_{k-k_{1}-j} \underbrace{t, \ldots, t}_{j}, \\
\underbrace{T, \ldots, T}_{n-k}) B(t)^{n-k_{1}} B\left(s_{1}\right) \cdots B\left(s_{k_{1}}\right)(\mathrm{d} s)^{\otimes k_{1}} .
\end{gathered}
$$

Since the number of variable $T$ is $n-k+k-k_{1}-j=n-k_{1}-j$, which does not depend on $k$, it enlightens us to change the 
order of summations. We want to sum over $k$ first. Observe that $\sum_{k=0}^{n} \sum_{k_{1}=0}^{k} \sum_{j=0}^{k-k_{1}}=\sum_{k_{1}=0}^{n} \sum_{j=0}^{n-k_{1}} \sum_{k=j+k_{1}}^{n}$; we obtain

$$
\begin{aligned}
\omega^{t} \circ A_{n}^{T}=\sum_{k_{1}=0}^{n} \sum_{j=0}^{n-k_{1}} \sum_{k=j+k_{1}}^{n}(-1)^{n-k}\left(\begin{array}{c}
n-k_{1}-j \\
n-k
\end{array}\right) \\
\quad \cdot \frac{(-1)^{n-j} n !}{k_{1} ! j !\left(n-k_{1}-j\right) !} \int_{[0, t]^{k_{1}}} \frac{\partial f_{n}}{\partial s_{1} \cdots \partial s_{k}}(s_{1}, \ldots, s_{k_{1}}, \underbrace{t, \ldots, t}_{j}, \underbrace{T, \ldots, T}_{n-k_{1}-j}) B(t)^{n-k_{1}} B\left(s_{1}\right) \cdots B\left(s_{k_{1}}\right)(\mathrm{d} s)^{\otimes k_{1}} .
\end{aligned}
$$

According to the property of binomial coefficient again

$$
\sum_{k=j+k_{1}}^{n}(-1)^{n-k}\left(\begin{array}{c}
n-k_{1}-j \\
n-k
\end{array}\right)=0 \quad \text { when } n>j+k_{1}
$$

We claim that (56) is not 0 only when $n=j+k_{1}$. Thus we have

$$
\begin{gathered}
\omega^{t} \circ A_{n}^{T}=\sum_{j+k_{1}=n}(-1)^{k_{1}} \\
\cdot\left(\begin{array}{c}
n \\
k_{1}
\end{array}\right) \int_{[0, t]^{k_{1}}} \frac{\partial f_{n}}{\partial s_{1} \cdots \partial s_{k}}(s_{1}, \ldots, s_{k_{1}}, \underbrace{t, \ldots, t}_{j}) \\
\cdot B(t)^{n-k_{1}} B\left(s_{1}\right) \cdots B\left(s_{k_{1}}\right)(\mathrm{d} s)^{\otimes k_{1}}=A_{n}^{t} .
\end{gathered}
$$

$$
\begin{aligned}
& \sum_{k=0}^{\lfloor n / 2\rfloor} \frac{n !}{2^{k}(n-2 k) !} \int_{t \leq u_{1} \leq \cdots \leq u_{k} \leq T} \omega^{t} \circ I_{n-2 k}\left(f_{n}\right)\left(u_{1}, u_{1}, \ldots, u_{k}, u_{k}\right)(\mathrm{d} u)^{\otimes k} \\
& =\sum_{k=0}^{\lfloor n / 2\rfloor} \sum_{k_{1}=0}^{\lfloor n / 2\rfloor-k} \int_{[t, T]^{k}} \int_{[0, T]^{k_{1}}} \frac{n !}{2^{k}(n-2 k) !} \frac{(-1)^{k_{1}}(n-2 k) !}{2^{k_{1}}\left(n-2 k-2 k_{1}\right) ! k_{1} !} A_{n-2 k-2 k_{1}}^{t}\left(u_{1}, u_{1}, \ldots, u_{k_{1}}, u_{k_{1}}, v_{1}, v_{1}, \ldots, v_{k}, v_{k}\right)(\mathrm{d} u)^{\otimes k_{1}}(\mathrm{~d} v)^{\otimes k}
\end{aligned}
$$

Let $m=k+k_{1}$ and we continue the above formula:

$$
\begin{aligned}
& =\sum_{k=0}^{\lfloor n / 2\rfloor} \sum_{m=k}^{\lfloor n / 2\rfloor} \int_{[t, T]^{k}} \int_{[0, T]^{m-k}} \frac{(-1)^{m} n !}{2^{m}(n-2 m) ! m !}(-1)^{k}\left(\begin{array}{c}
m \\
k
\end{array}\right) A_{n-2 m}^{t}\left(u_{1}, u_{1}, \ldots, u_{m-k}, u_{m-k}, v_{1}, v_{1}, \ldots, v_{k}, v_{k}\right)(\mathrm{d} u)^{\otimes m-k}(\mathrm{~d} v)^{\otimes k} \\
& =\sum_{m=0}^{\lfloor n / 2\rfloor} \sum_{k=0}^{m} \int_{[t, T]^{k}} \int_{[0, T]^{m-k}} \frac{(-1)^{m} n !}{2^{m}(n-2 m) ! m !}(-1)^{k}\left(\begin{array}{c}
m \\
k
\end{array}\right) A_{n-2 m}^{t}\left(u_{1}, u_{1}, \ldots, u_{m-k}, u_{m-k}, v_{1}, v_{1}, \ldots, v_{k}, v_{k}\right)(\mathrm{d} u)^{\otimes m-k}(\mathrm{~d} v)^{\otimes k} .
\end{aligned}
$$

tep 3. Now we can prove recurrence formula (10).

By (52) and (42), we have

$$
\begin{gathered}
I_{n}\left(f_{n} \chi_{[0, t]}\right)=\sum_{k=0}^{\lfloor n / 2\rfloor} \frac{(-1)^{k} n !}{2^{k}(n-2 k) ! k !} \\
\cdot \int_{[0, t]^{k}} A_{n-2 k}^{t}\left(u_{1}, u_{1}, \ldots, u_{k}, u_{k}\right)(\mathrm{d} u)^{\otimes k} ; \\
\omega^{t} \circ I_{n}\left(f_{n}\right)=\sum_{k=0}^{\lfloor n / 2\rfloor} \frac{(-1)^{k} n !}{2^{k}(n-2 k) ! k !} \\
\cdot \int_{[0, T]^{k}} A_{n-2 k}^{t}\left(u_{1}, u_{1}, \ldots, u_{k}, u_{k}\right)(\mathrm{d} u)^{\otimes k} .
\end{gathered}
$$

Now we apply another basic rule of integration, for a $m$ variable symmetric function $g_{m}$

$$
\int_{[0, t]^{m}} g_{m}(\mathrm{~d} u)^{\otimes m}=\int_{([0, T] \backslash[t, T])^{m}} g_{m}(\mathrm{~d} u)^{\otimes m}=\sum_{k=0}^{m} \int_{[t, T]^{k}} \int_{[0, T]^{m-k}}(-1)^{k}\left(\begin{array}{l}
m \\
k
\end{array}\right) g_{m}\left(u_{1}, \ldots, u_{m-k}, v_{1}, \ldots, v_{k}\right)(\mathrm{d} u)^{\otimes(m-k)}(\mathrm{d} v)^{\otimes k}
$$


Now apply (62) in (61) and we finally obtain

$$
\begin{aligned}
& \sum_{k=0}^{\lfloor n / 2\rfloor} \frac{n !}{2^{k}(n-2 k) !} \int_{t \leq u_{1} \leq \cdots \leq u_{k} \leq T} \omega^{t} \circ I_{n-2 k}\left(f_{n}\right)\left(u_{1}, u_{1}, \ldots,\right. \\
& \left.u_{k}, u_{k}\right)(\mathrm{d} u)^{\otimes k} \\
& \quad=\sum_{m=0}^{\lfloor n / 2\rfloor} \int_{[0, t]^{m}} \frac{(-1)^{m} n !}{2^{m}(n-2 m) ! m !} A_{n-2 m}^{t}\left(u_{1}, u_{1}, \ldots, u_{m},\right. \\
& \left.u_{m}\right)(\mathrm{d} u)^{\otimes m}=I_{n}\left(f_{n} \chi_{[0, t]}\right) .
\end{aligned}
$$

Step 4. We now use induction to prove (11), based on (10). For simplicity, we introduce

$$
\begin{aligned}
& a_{n-2 k} \\
& \quad:=\int_{[t, T]^{k}} \omega^{t} \circ I_{n-2 k}\left(f_{n}\right)\left(u_{1}, u_{1}, \ldots, u_{k}, u_{k}\right)(\mathrm{d} u)^{\otimes k} ; \\
& b_{n-2 k} \\
& \quad:=\int_{[t, T]^{k}} I_{n-2 k}\left(f_{n} \chi_{[0, t]}\right)\left(u_{1}, u_{1}, \ldots, u_{k}, u_{k}\right)(\mathrm{d} u)^{\otimes k}
\end{aligned}
$$

for $k \leq\lfloor n / 2\rfloor$. Then (10) implies

$$
a_{n}=b_{n}-\sum_{k=1}^{\lfloor n / 2\rfloor} \frac{n !}{2^{k}(n-2 k) ! k !} a_{n-2 k}
$$

We calculate the right hand side of (11) with (65): let $m=k+k_{1}$

$$
\begin{aligned}
& n ! \sum_{k=0}^{\lfloor n / 2\rfloor} \frac{(-1)^{k}}{2^{k}(n-2 k) ! k !} b_{n-2 k} \\
& \quad=n ! \sum_{k=0}^{\lfloor n / 2\rfloor} \sum_{k_{1}=0}^{\lfloor n / 2\rfloor-k} \frac{(-1)^{k}}{2^{k}(n-2 k) ! k !} \\
& \cdot \frac{(n-2 k) !}{2^{k_{1}}\left(n-2 k-2 k_{1}\right) ! k_{1} !} a_{n-2 k-2 k_{1}} \\
& =\sum_{k=0}^{\lfloor n / 2\rfloor} \sum_{k_{1}=0}^{\lfloor n / 2\rfloor-k} \frac{(-1)^{k} n !}{2^{k+k_{1}}\left(n-2\left(k+k_{1}\right)\right) ! k_{1} ! k !} a_{n-2 k-2 k_{1}}
\end{aligned}
$$

$$
\begin{aligned}
& =\sum_{m=0}^{\lfloor n / 2\rfloor} \frac{(-1)^{m} n !}{2^{m}(n-2 m) ! m !} a_{n-2 m} \sum_{k_{1}=0}^{m}(-1)^{k_{1}}\left(\begin{array}{l}
m \\
k_{1}
\end{array}\right)=a_{n} \\
& =\omega^{t} \circ I_{n}\left(f_{n}\right) .
\end{aligned}
$$

The proposition is proved.

3.5. Proof of Theorem 3. The proof is constructive. For any fixed $t \in[0, T]$, if $F$ has its chaos decomposition $\sum_{n=0}^{\infty} I_{n}\left(f_{n}\right)$, then for fixed $N$ (depending on $M$ ), we will study $F^{M, N}$ := $\sum_{n=0}^{M} I_{n}\left(f_{n}^{N}\right)$, where

$$
\begin{aligned}
& f_{n}^{N}\left(s_{1}, \ldots, s_{n}\right):=f_{n}\left(t \chi_{[s, s+1 / N]}\left(s_{1}\right)\right. \\
& \quad+s_{1} \chi_{[0, T] \backslash[s, s+1 / N]}\left(s_{1}\right), \ldots, t \chi_{[s, s+1 / N]}\left(s_{n}\right) \\
& \left.\quad+s_{n} \chi_{[0, T] \backslash[s, s+1 / N]}\left(s_{n}\right)\right) .
\end{aligned}
$$

In other words, the kernel $f_{n}^{N}$ is constant when its arguments lie between $s$ and $s+1 / N$. Then we have the following lemma.

Lemma 12. $\omega^{t} \circ I_{n}\left(f_{n}^{N}\right) \underset{N \rightarrow \infty}{\stackrel{L^{2}(\mathbb{P})}{\longrightarrow}} \omega^{t} \circ I_{n}\left(f_{n}\right)$ and in particular

$$
E\left[\left(\omega^{t} \circ I_{n}\left(f_{n}^{N}\right)-I_{n}\left(f_{n}\right)\right)^{2}\right] \leq \frac{C(n !)^{2} n^{7}}{N^{3}},
$$

where $C$ is a constant which does not depend on $N$ and $n$.

Proof. For any fixed $n$, we define a sequence of sets $\left\{A_{k_{1}, k_{2}}\right\}_{k_{1}+k_{2} \leq n}$ as

$$
\begin{gathered}
A_{k_{1}, k_{2}}:=\left\{s_{1}, \ldots, s_{n}: 0 \leq s_{1} \leq \cdots \leq s_{k_{1}} \leq t \leq s_{k_{1}+1}\right. \\
\left.\leq \cdots \leq s_{k_{1}+k_{2}} \leq t+\frac{1}{N} \leq s_{k_{1}+k_{2}+1} \leq \cdots \leq s_{n} \leq T\right\} .
\end{gathered}
$$

Observe that on $A_{k_{1}, 0}$ the kernels $f_{n}$ and $f_{n}^{N}$ coincide. According to (67), we obtain

$$
\begin{aligned}
& \omega^{t} \circ I_{n}\left(f_{n}\right)-\omega^{t} \circ I_{n}\left(f_{n}^{N}\right) \\
& =n ! \sum_{k_{1}+k_{2} \leq n, k_{2} \neq 0} \omega^{t} \\
& \quad \circ \int_{A_{k_{1}, k_{2}}}\left(f_{n}-f_{n}^{N}\right)\left(s_{1}, \ldots, s_{n}\right)(\mathrm{d} B(s))^{\otimes n} .
\end{aligned}
$$

To bound (70), we apply Proposition 2 to obtain

$$
E\left[\left(\omega^{t} \circ I_{n}\left(f_{n}\right)\right)^{2}\right]=(n !)^{2} \sum_{k=0}^{n} \frac{1}{(k !)^{2}} \int_{[t, T]^{k}} \int_{\left\{0 \leq s_{1} \leq \cdots \leq s_{n-k} \leq t\right\}} f_{n}\left(s_{1}, \ldots, s_{n-k}, u_{1}, \ldots, u_{k}\right)^{2}(\mathrm{~d} s)^{\otimes n-k}(\mathrm{~d} u)^{\otimes k}<\infty
$$


Now we apply (71) on (70) and by Cauchy-Schwartz inequality, we have

$$
\begin{gathered}
E\left[\left(\omega^{t} \circ I_{n}\left(f_{n}^{N}\right)-\omega^{t} \circ I_{n}\left(f_{n}\right)\right)^{2}\right] \leq(n n !)^{2} \sum_{k_{1}+k_{2} \leq n, k_{2} \neq 0} E\left[\left(\omega^{t} \circ \int_{A_{k_{1}, k_{2}}}\left(f_{n}-f_{n}^{N}\right)\left(s_{1}, \ldots, s_{n}\right)(\mathrm{d} B(s))^{\otimes n}\right)^{2}\right]=(n n !)^{2} \\
\quad \sum_{k_{1}+k_{2} \leq n, k_{2} \neq 0} \sum_{k=n-k_{1}}^{n} \frac{1}{(k !)^{2}} \int_{[t, T]^{k}} \int_{\left\{0 \leq s_{1} \leq \cdots \leq s_{n-k} \leq t\right\}}\left(f_{n}-f_{n}^{N}\right)\left(s_{1}, \ldots, s_{n-k}, u_{1}, \ldots, u_{k}\right)^{2} \chi_{A_{k_{1}, k_{2}}}\left(s_{1}, \ldots, s_{n}\right)(\mathrm{d} s)^{\otimes n-k}(\mathrm{~d} u)^{\otimes k} .
\end{gathered}
$$

Since $f_{n}$ is differentiable with respect to $s_{1}, \ldots, s_{n}$, there exists a constant $C_{n}$ such that

$$
\begin{aligned}
& \left|f_{n}\left(s_{1}, x_{1}, \ldots, s_{n}, x_{n}\right)-f_{n}\left(t, x_{1}, \ldots, t, x_{n}\right)\right| \\
& \quad \leq C_{n} n\left(\sup _{s_{1}, \ldots, s_{n}}\left(s_{i}-t\right)\right) .
\end{aligned}
$$

Therefore following (72), we obtain

$$
E\left[\left(\omega^{t} \circ I_{n}\left(f_{n}^{N}\right)-\omega^{t} \circ I_{n}\left(f_{n}\right)\right)^{2}\right] \leq \frac{C(n n !)^{2} n^{5}}{N^{3}},
$$

where $C$ is a constant which does not depend on $n$ and $N$.

Now we construct $F^{N}$ by $\sum_{n=0}^{\infty} I_{n}\left(f_{n}^{N}\right)$. To prove the theorem, we introduce two subseries $F^{M, N}$ and $F^{M}$ by

$$
\begin{aligned}
F^{M, N} & :=\sum_{n=0}^{M} I_{n}\left(f_{n}^{N}\right) \underset{L^{2}(\mathbb{P})}{\stackrel{M \rightarrow \infty}{\longrightarrow}} F^{N} ; \\
F^{M} & :=\sum_{n=0}^{M} I_{n}\left(f_{n}\right) \underset{L^{2}(\mathbb{P})}{\stackrel{M \rightarrow \infty}{\longrightarrow}} F .
\end{aligned}
$$

For enough large $N$, we choose $M$ such that $\left(M^{7}(M !)^{2}\right)^{1 / 3} M \leq N$. Then by Lemma 12 and CauchySchwarz inequality, there exists a constant $\varepsilon \in(0,1)$ such that

$$
\begin{aligned}
& E\left[\left(\omega^{t} \circ\left(F^{M, N}-F^{M}\right)\right)^{2}\right] \\
& =E\left[\left(\sum_{n=0}^{M}\left(\omega^{t} \circ I_{n}\left(f_{n}\right)-\omega^{t} \circ I_{n}\left(f_{n}^{N}\right)\right)\right)^{2}\right] \\
& \leq C M\left(\sum_{n=0}^{M} \frac{(n n !)^{2} n^{5}}{N^{3}}\right) \leq \frac{C}{N^{2+\varepsilon}} .
\end{aligned}
$$

Then using triangle inequality, we prove the theorem.
3.6. Proof of Theorem 4. For any $F \in L^{2}(\mathbb{P}), s \in[t, T]$, we choose the sequence $\left\{F^{N}\right\}_{N \geq 0}$ constructed in Theorem 3 . Then by the Clark-Ocone formula, we obtain

$$
\begin{aligned}
E & {\left[F^{N} \mid \mathscr{F}_{s-1 / N}\right] } \\
= & E\left[F^{N} \mid \mathscr{F}_{s}\right]-\int_{s-1 / N}^{s} E\left[\mathbb{D}_{s} F^{N} \mid \mathscr{F}_{s}\right] \mathrm{d} B\left(s_{1}\right) \\
& +\int_{s-1 / N}^{s} \int_{s_{1}}^{s} E\left[\mathbb{D}_{s}^{2} F^{N} \mid \mathscr{F}_{s}\right] \mathrm{d} B\left(s_{2}\right) \mathrm{d} B\left(s_{1}\right) \\
& -R_{[s-1 / N, s]}^{3},
\end{aligned}
$$

where

$$
\begin{aligned}
& R_{[s-1 / N, s]}^{3} \\
& =\int_{s-1 / N}^{s} \int_{s_{1}}^{s} \int_{s_{2}}^{s} E\left[\mathbb{D}_{s}^{3} F^{N} \mid \mathscr{F}_{s_{3}}\right] \mathrm{d} B\left(s_{3}\right) \mathrm{d} B\left(s_{2}\right) \mathrm{d} B\left(s_{1}\right) .
\end{aligned}
$$

On one hand, by Lemma 5.2 in [5], we obtain

$$
\begin{aligned}
& E\left[\left(R_{[s-1 / N, s]}^{3}\right)^{2}\right] \\
& \quad \leq \sum_{i=0}^{3} E\left[\left(\mathbb{D}_{s}^{6-i} F^{N}\right)^{2}\right]\left(\begin{array}{l}
3 \\
i
\end{array}\right)^{4} \frac{i !}{(3 !)^{2}} \frac{1}{N^{6-i}}
\end{aligned}
$$

On the other hand, we can compute

$$
\begin{aligned}
\omega^{t} \circ\left(-\int_{s-1 / N}^{s} E\left[\mathbb{D}_{s} F^{N} \mid \mathscr{F}_{s}\right] \mathrm{d} B\left(s_{1}\right)\right) \\
=\frac{1}{N} \omega^{t} \circ E\left[\mathbb{D}_{s}^{2} F^{N} \mid \mathscr{F}_{s}\right] ; \\
\omega^{t} \circ\left(\int_{s-1 / N}^{s} \int_{s_{1}}^{s} E\left[\mathbb{D}_{s}^{2} F^{N} \mid \mathscr{F}_{s}\right] \mathrm{d} B\left(s_{2}\right) \mathrm{d} B\left(s_{1}\right)\right) \\
=\omega^{t} \\
\quad \circ\left(-\frac{1}{2 N} E\left[\mathbb{D}_{s}^{2} F^{N} \mid \mathscr{F}_{s}\right]+\frac{1}{2 N^{2}} E\left[\mathbb{D}_{s}^{4} F^{N} \mid \mathscr{F}_{s}\right]\right) .
\end{aligned}
$$


Then we can establish the equation as

$$
\begin{aligned}
& E\left[\left(\omega^{t} \circ N\left(E\left[F^{N} \mid \mathscr{F}_{s-1 / N}\right]-E\left[F^{N} \mid \mathscr{F}_{s}\right]\right)+\omega^{t}\right.\right. \\
& \left.\left.\quad \circ \frac{1}{2} \mathbb{D}_{s}^{2} E\left[F^{N} \mid \mathscr{F}_{s}\right]\right)^{2}\right] \leq 2 E\left[\left(\omega^{t}\right.\right. \\
& \left.\left.\quad \circ \frac{1}{2 N} E\left[\mathbb{D}_{s}^{4} F^{N} \mid \mathscr{F}_{s}\right]\right)^{2}\right]+2 E\left[\left(\omega^{t} \circ R_{[s-1 / N, s]}^{3}\right)^{2}\right] \\
& \quad=O\left(\frac{1}{N^{2}}\right),
\end{aligned}
$$

where the last equality follows from (79), Proposition 2. Thus combining (77), (79), (80), and (81) as well as the assumption $F \in \mathbb{D}^{6}([0, T])$ and Proposition 2, we have

$$
\begin{gathered}
\omega^{t} \circ\left(N\left(E\left[F^{N} \mid \mathscr{F}_{s-1 / N}\right]-E\left[F^{N} \mid \mathscr{F}_{s}\right]\right)\right. \\
\left.-\frac{1}{2} \mathbb{D}_{s}^{2} E\left[F^{N} \mid \mathscr{F}_{s}\right]\right) \underset{N \rightarrow \infty}{\stackrel{L^{2}(\mathbb{P})}{\longrightarrow}} 0 .
\end{gathered}
$$

Here, for simplicity, we define $L^{2}$ norm $\|\cdot\|_{L^{2}(\mathbb{P})}^{2}:=E\left[(\cdot)^{2}\right]$. Then, from Theorem 3 and the closability of the Malliavin derivative operator, for some constant $\varepsilon<1$,

$$
\begin{aligned}
& \left\|\omega^{t} \circ E\left[\mathbb{D}_{s} F^{N} \mid \mathscr{F}_{s}\right]-\omega^{t} \circ E\left[\mathbb{D}_{s} F \mid \mathscr{F}_{s}\right]\right\|_{L^{2}(\mathbb{P})}^{2} \\
& \quad \leq \frac{C}{N^{2+\varepsilon}} .
\end{aligned}
$$

With triangle inequality and Cauchy-Schwartz inequality, we finally have, using (81) and (83),

$$
\begin{aligned}
& \| \omega^{t} \circ N\left(E\left[F \mid \mathscr{F}_{s-1 / N}\right]-E\left[F \mid \mathscr{F}_{s}\right]\right)-\omega^{t} \circ \frac{1}{2} \\
& \cdot \mathbb{D}_{s}^{2} E\left[F \mid \mathscr{F}_{s}\right]\left\|_{L^{2}(\mathbb{P})}^{2} \leq\right\| \omega^{t} \circ N\left(E\left[F \mid \mathscr{F}_{s-1 / N}\right]\right. \\
& \left.-E\left[F^{N} \mid \mathscr{F}_{s-1 / N}\right]\right)\left\|_{L^{2}(\mathbb{P})}^{2}+\right\| \omega^{t} \circ N\left(E\left[F \mid \mathscr{F}_{s}\right]\right. \\
& \left.-E\left[F^{N} \mid \mathscr{F}_{s}\right]\right)\left\|_{L^{2}(\mathbb{P})}^{2}+\right\| \omega^{t} \\
& \circ\left(N\left(E\left[F^{N} \mid \mathscr{F}_{s}\right]-E\left[F^{N} \mid \mathscr{F}_{s-1 / N}\right]\right)-\omega^{t}\right. \\
& \left.\circ \frac{1}{2} \mathbb{D}_{s}^{2} E\left[F^{N} \mid \mathscr{F}_{s}\right]\right)\left\|_{L^{2}(\mathbb{P})}^{2}+\right\| \omega^{t} \circ \frac{1}{2} \\
& \cdot \mathbb{D}_{s}^{2} E\left[F^{N} \mid \mathscr{F}_{s}\right]-\omega^{t} \circ \frac{1}{2} \mathbb{D}_{s}^{2} E\left[F \mid \mathscr{F}_{s}\right] \|_{L^{2}(\mathbb{P})}^{2} \\
& \leq \frac{C}{N^{\varepsilon}}
\end{aligned}
$$

or in other words

$$
\frac{\mathrm{d} \omega^{t} \circ E\left[F \mid \mathscr{F}_{s}\right]}{\mathrm{d} s}=-\omega^{t} \circ \frac{1}{2} \mathbb{D}_{s}^{2} E\left[F \mid \mathscr{F}_{s}\right] .
$$

3.7. Proof of Theorem 6. For $i=1, \ldots, N(T-s)$, define

$$
\begin{aligned}
x_{i}^{N} & :=N \omega^{t} \circ\left(E\left[F \mid \mathscr{F}_{s+(i-1) / N}\right]-E\left[F \mid \mathscr{F}_{s+i / N}\right]\right. \\
& \left.-\frac{1}{2 N} \mathbb{D}_{s+1 / N}^{2} E\left[F \mid \mathscr{F}_{s+i / N}\right]\right) .
\end{aligned}
$$

We rewrite (84) as

$$
E\left[\left(\frac{x_{i}^{N}}{N}\right)^{2}\right] \leq \frac{C}{N^{2+\varepsilon}}
$$

Jensen's inequality states that

$$
\sum_{i=1}^{N} E\left[\left(\frac{x_{i}^{N}}{N}\right)^{2}\right] \leq \frac{\sum_{i=1}^{N} E\left[\left(x_{i}^{N}\right)^{2}\right]}{N} \leq \frac{C}{N^{\varepsilon}} .
$$

Since $\int_{s}^{T}(1 / 2) \mathbb{D}_{u}^{2} E\left[F \mid \mathscr{F}_{u}\right] \mathrm{d} u$ is bounded in $L^{2}(\mathbb{P})$, then

$$
\begin{aligned}
& \sum_{i=1}^{N} \frac{x_{i}^{N}}{N} \underset{L^{2}(\mathbb{P})}{\stackrel{N \rightarrow \infty}{\longrightarrow}} \omega^{t} \\
& \quad \circ\left(E\left[F \mid \mathscr{F}_{s}\right]-F-\int_{s}^{T} \frac{1}{2} \mathbb{D}_{u}^{2} E\left[F \mid \mathscr{F}_{u}\right] \mathrm{d} u\right) .
\end{aligned}
$$

Using (88), we thus proved that, in $L^{2}(\mathbb{P})$,

$$
\begin{aligned}
\omega^{t} \circ E\left[F \mid \mathscr{F}_{s}\right]= & \omega^{t} \circ F \\
& +\int_{s}^{T} \omega^{t} \circ \frac{1}{2} \mathbb{D}_{s}^{2} E\left[F \mid \mathscr{F}_{u}\right] \mathrm{d} u .
\end{aligned}
$$

Then for positive integer $n$ we define the operator $T_{s}^{(n)}$ by

$$
T_{s}^{(n)} F:=\sum_{i=0}^{n} \mathscr{A}_{i, s} F
$$

where

$$
\mathscr{A}_{i, s} F:=\int_{s \leq s_{1} \leq \cdots \leq s_{i} \leq T} \frac{1}{2^{i}} \mathbb{D}_{s_{1}}^{2} \cdots \mathbb{D}_{s_{i}}^{2} F(\mathrm{~d} s)^{\otimes i} .
$$

Then by iterating (90) we obtain the following: for $n>0$

$$
\begin{gathered}
\omega^{t} \circ E\left[F \mid \mathscr{F}_{s}\right]=\omega^{t} \circ\left(T_{s}^{(n-1)} F\right)+\frac{1}{2^{n}} \\
\cdot \int_{s \leq u_{1} \leq \cdots \leq u_{n} \leq T} \omega^{t} \circ \mathbb{D}_{s_{1}}^{2} \cdots \mathbb{D}_{s_{n}}^{2} E\left[F \mid \mathscr{F}_{u_{n}}\right](\mathrm{d} u)^{\otimes n} .
\end{gathered}
$$

Thus according to Condition 1,

$$
\begin{aligned}
& E\left[\left(\omega^{t} \circ\left(\left(E_{s}-T_{s}^{(n-1)}\right) F\right)\right)^{2}\right]=E\left[\left(\frac{1}{2^{n}}\right.\right. \\
& \quad \cdot \int_{s \leq u_{1} \leq \cdots \leq u_{n} \leq T} \omega^{t} \\
& \left.\left.\quad \circ \mathbb{D}_{s_{1}}^{2} \cdots \mathbb{D}_{s_{n}}^{2} E\left[F \mid \mathscr{F}_{u_{n}}\right](\mathrm{d} u)^{\otimes n}\right)^{2}\right] \leq \frac{(T-s)^{2 n}}{\left(2^{n} n !\right)^{2}}
\end{aligned}
$$

$$
\cdot E\left[\sup _{u_{1}, \ldots, u_{n} \in[0, T]}\left|\omega^{t} \circ \mathbb{D}_{s_{1}}^{2} \cdots \mathbb{D}_{s_{n}}^{2} F\right|^{2}\right] \underset{n \rightarrow \infty}{\longrightarrow} 0 .
$$


We now take $s=t$ and obtain

$$
\begin{aligned}
E & {\left[F \mid \mathscr{F}_{t}\right]=E_{t} F=\omega^{t} \circ\left(T_{t}^{(\infty)} F\right) } \\
& =\sum_{n=0}^{\infty} \frac{1}{2^{n}} \int_{t \leq u_{1} \leq \cdots \leq u_{n} \leq T} \omega^{t} \circ \mathbb{D}_{s_{1}}^{2} \cdots \mathbb{D}_{s_{n}}^{2} F(\mathrm{~d} u)^{\otimes n} .
\end{aligned}
$$

\section{Competing Interests}

The authors declare that there is no conflict of interests regarding the publication of this paper.

\section{References}

[1] B. Dupire, "Functional Ito calculus," Portfolio Research Paper 2009-04, Bloomberg, 2009.

[2] R. Cont and D.-A. Fournie, "Functional Ito calculus and stochastic integral representation of martingales," The Annals of Probability, vol. 41, no. 1, pp. 109-133, 2013.

[3] R. McOwen, Partial Differential Equations, Methods and Applications, Prentice Hall, 1996.

[4] K. Yosida, Functional Analysis, Springer, 1978.

[5] S. Jin, Q. Peng, and H. Schellhorn, "A representation theorem for smooth Brownian martingales," Stochastics, vol. 88, no. 5, pp. 651-679, 2016.

[6] S. Peng and F. Wang, "BSDE, path-dependent PDE and nonlinear Feynman-Kac formula," Science China Mathematics, vol. 59, no. 1, pp. 19-36, 2016.

[7] I. Ekren, C. Keller, N. Touzi, and J. Zhang, "On viscosity solutions of path dependent PDEs," The Annals of Probability, vol. 42, no. 1, pp. 204-236, 2014.

[8] D. Nualart, The Malliavin Calculus and Related Topics, Springer, 1995.

[9] J. Yong and X. Y. Zhou, Stochastic Controls: Hamiltonian Systems and HJB Equations, vol. 43 of Applications of Mathematics (New York), Springer, New York, NY, USA, 1999. 


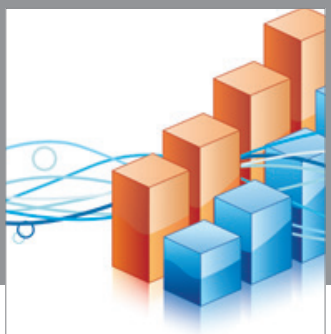

Advances in

Operations Research

vatem alat4

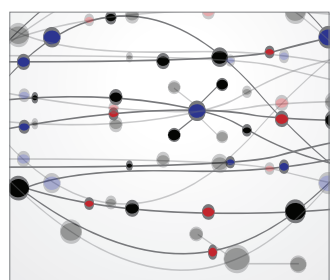

\section{The Scientific} World Journal
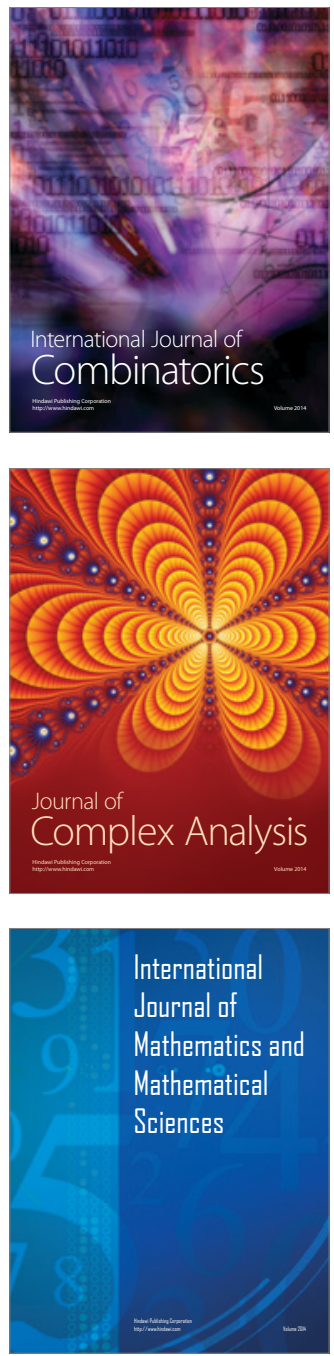
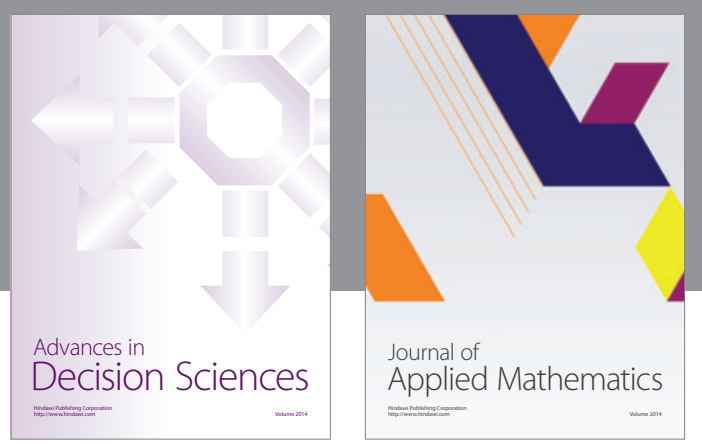

Algebra

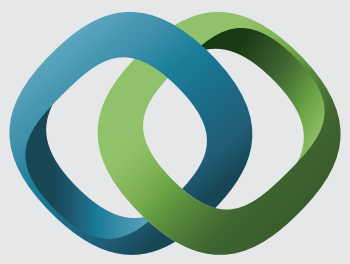

\section{Hindawi}

Submit your manuscripts at

https://www.hindawi.com
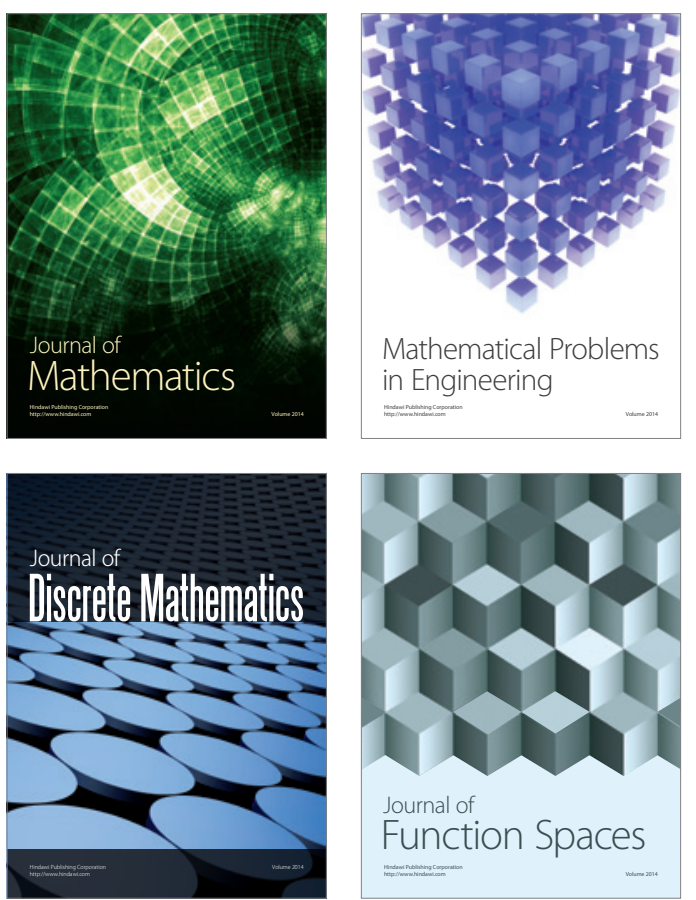

Mathematical Problems in Engineering
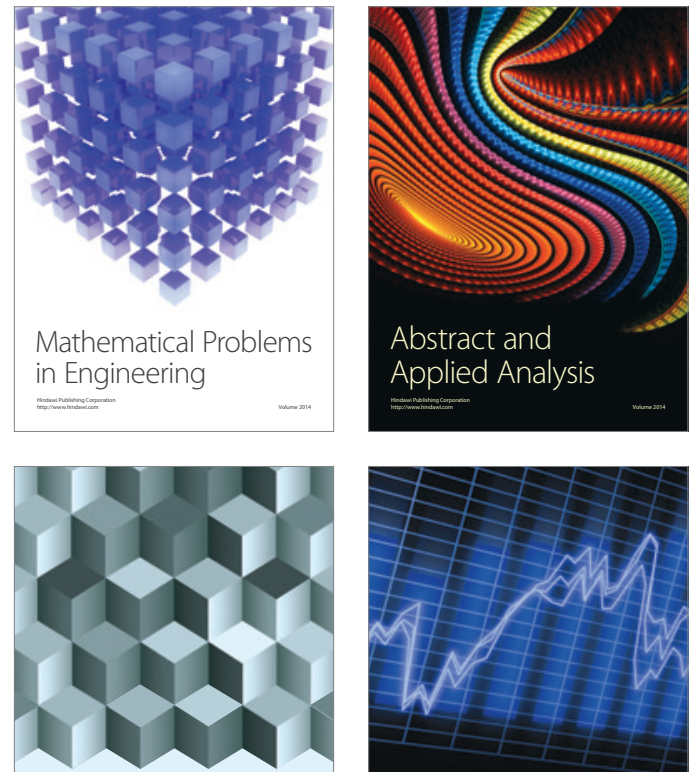

Journal of

Function Spaces

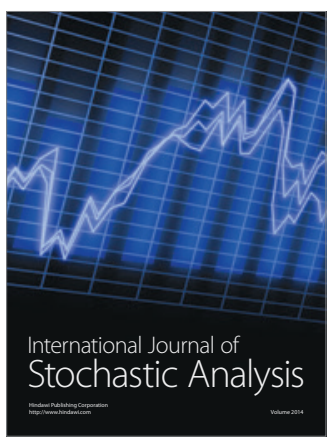

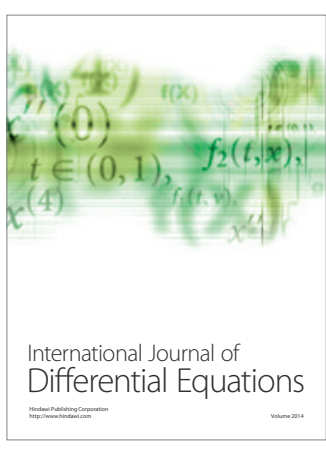
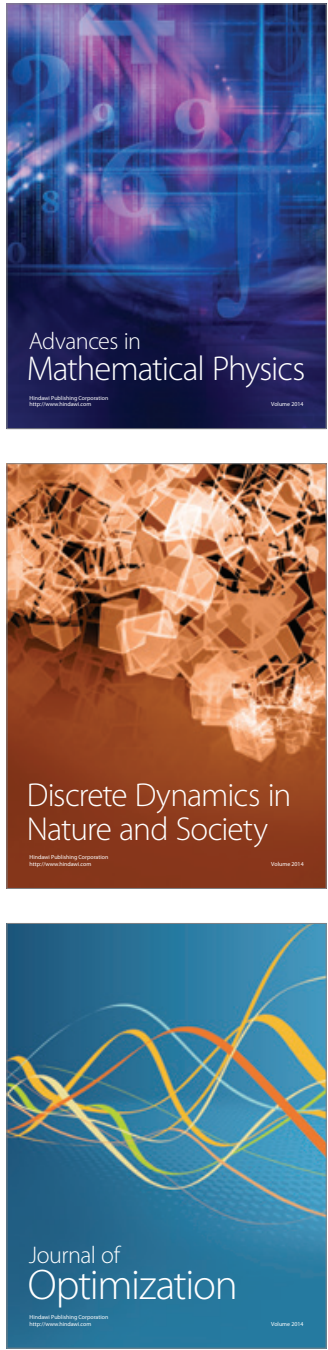